\title{
ON THE WEAKEST FAILURE DETECTOR FOR NON-BLOCKING ATOMIC COMMIT*
}

\author{
Rachid Guerraoui \\ Petr Kouznetsov \\ Distributed Programming Laboratory \\ Swiss Federal Institute of Technology in Lausanne
}

\begin{abstract}
This paper addresses the question of the weakest failure detector to solve the Non-Blocking Atomic Commit (NBAC) problem in an asynchronous system. We define the set $\mathcal{A}$ of timeless failure detectors which excludes failure detectors that provide information about global time but includes all known meaningful failure detectors such as $\diamond \mathcal{S}, \diamond \mathcal{P}$ and $\mathcal{P}$ [2]. We show that, within $\mathcal{A}$, the weakest failure detector for NBAC is ? $\mathcal{P}+\diamond \mathcal{S}$.
\end{abstract}

\section{Introduction}

Problem. Non-Blocking Atomic Commit (NBAC) is a typical agreement problem in distributed computing [9]. To ensure the atomicity of a distributed transaction, the processes must agree on a common outcome: commit or abort. Every process that does not crash during the execution of the algorithm (i.e., a correct process), should eventually decide on an outcome without waiting for crashed processes to recover.

More precisely, the NBAC problem consists for a set of processes to reach a common decision, commit or abort, according to some initial votes of the processes, yes or no, such that the following properties are satisfied: (1) Agreement: No two processes decide differently; (2) Termination: Every correct process eventually decides; (3) A-Validity: Abort is the only possible decision if some process votes no; and (4) C-Validity: Commit is the only possible decision if all processes are correct and vote yes.

In this paper, we discuss the solvability of the problem in a crash-stop asynchronous message-passing model of distributed computing. Informally, the model is one in which processes exchange messages through reliable commu-

*This work is partially supported by the Swiss National Science Foundation (project number 510-207). 
nication channels, processes can fail by crashing, and there are no bounds on message transmission time and relative processor speeds.

Background. It is well-known that many fundamental agreement problems in distributed computing, in particular, the well-known Consensus problem, cannot be solved deterministically in an asynchronous system that is subject to even a single crash failure [3]. In Consensus, the processes need to decide on one out of two values, 0 or 1 , based on proposed values, 0 or 1 , so that, in addition to the Agreement and Termination properties of NBAC, the following Validity property holds: A value decided must be a value proposed.

To circumvent the impossibility of Consensus, Chandra and Toueg [2] introduced the notion of failure detector. Informally, a failure detector is a distributed oracle that gives (possibly incorrect) hints about the crashes of processes. Each process has access to a local failure detector module that monitors other processes in the system. In [2], it is shown that a rather weak failure detector $\diamond \mathcal{S}$, called the eventually strong failure detector, is sufficient to solve Consensus in an asynchronous system with a majority of correct processes. It is shown in [1] that and failure detector that solves Consensus can emulate $\diamond \mathcal{S}$ : hence $\diamond \mathcal{S}$ is the weakest failure detector to solve the problem. In other words, $\diamond \mathcal{S}$ encapsulates the exact information about failures needed to solve Consensus in a system with a majority of correct processes.

Like Consensus, NBAC does not admit a deterministic solution in an asynchronous system even in the face of a single failure. In this paper we focus on the question of the weakest failure detector to solve NBAC.

Conjecture: ?P $+\diamond \mathcal{S}$. Guerraoui introduced in [6] the anonymously perfect failure detector ?P and showed that ?P is necessary to solve NBAC. Each module of ?P at a given process outputs either the empty set or the identifier of the process. When the failure detector module of ?P at a process $p_{i}$ outputs $p_{i}$, we say that $p_{i}$ detects a crash. ?P satisfies the following properties: Anonymous Completeness: If some process crashes, then there is a time after which every correct process permanently detects a crash, and Anonymous Accuracy: No crash is detected unless some process crashes.

In other words, ?P correctly detects that some process has crashed, but does not tell which process has actually crashed. An algorithm that transforms Consensus into NBAC using ?P is presented in [6]. Since $\diamond \mathcal{S}$ is sufficient to solve Consensus in a system with a majority of correct processes, ?P $+\diamond \mathcal{S}$ is sufficient to solve NBAC in this environment. It is also shown in [6] that $? \mathcal{P}+\diamond \mathcal{S}$ is strictly weaker than the Perfect failure detector $\mathcal{P}$. Evidently, $\diamond \mathcal{S}$ is strictly weaker than ?P$+\diamond \mathcal{S}$.

The conjecture we want to prove is that ?P $+\diamond \mathcal{S}$ is the weakest failure detector to solve NBAC (with a majority of correct processes). To show this, we need to prove that any algorithm that solves NBAC can be used to emulate $\diamond \mathcal{S}$. 
Assumptions. If we consider the overall universe of failure detectors defined in [2], $\diamond \mathcal{S}$ is not necessary to solve NBAC (i.e., our conjecture is not true). Indeed, Guerraoui introduced in [6] a stillborn failure detector, denoted by $\mathcal{B}$, that solves NBAC and cannot be transformed into ?P $+\diamond \mathcal{S}[6]$. More precisely, $\mathcal{B}$ ensures that every initial crash is immediately detected by every process $p_{i}$, so that $p_{i}$ can safely decide abort without synchronizing with others processes.

More generally, any failure detector that tells the time when a failure occurred can solve NBAC without employing Consensus. Consider a failure detector $\mathcal{B}[\alpha]$, such that at each process $p_{i}, \mathcal{B}[\alpha]$ outputs a singleton $T$ until some time $t_{i}$. At time $t_{i}$, if some process is crashed at time $\alpha$, the failure detector module outputs $p_{i}$. Otherwise, after $t_{i}, \mathcal{B}[\alpha]$ behaves like $\mathcal{P}$ (the perfect failure detector). It can be easily shown that $\mathcal{B}[\alpha]$ is not transformable into $\diamond \mathcal{S}$, although it solves NBAC as follows: each process $p_{i}$ decides abort whenever its failure detector module outputs $p_{i}$ instead of $\mathrm{T}$, otherwise $p_{i}$ runs the $3 \mathrm{PC}$ algorithm [9]. However, $\mathcal{B}[\alpha]$ reports the exact time when a failure occurred, which can be provided only through the global time source. We question ourselves what happens if we rule out time-based failure detectors like $\mathcal{B}[\alpha]$ : among the remaining failure detectors, is ?P $+\diamond \mathcal{S}$ indeed the weakest to solve NBAC?

Contributions. This paper shows that the answer is "yes", i.e., our initial conjecture is true under the assumption that failure detectors do not reveal time.

We define a new class $\mathcal{A}$ of timeless failure detectors (restricting the original universe of failure detectors of [2]) that excludes time-based failure detectors like $\mathcal{B}[\alpha]$, but includes all known failure detectors like $\mathcal{P}, \diamond \mathcal{S}$ and ?P. Informally, a timeless failure detector module is not able to provide information about when exactly (in the sense of the global time) failures have occurred.

We show that in $\mathcal{A}, \diamond \mathcal{S}$ is necessary to solve NBAC. That is, any failure detector of $\mathcal{A}$ that solves NBAC can emulate $\diamond \mathcal{S}$. To show this, we extend the technique used in [1] to prove that $\diamond \mathcal{S}$ is necessary to solve Consensus [1]. This extension is not trivial. Given that no information about time can be provided by a timeless failure detector, for any execution scenario, we construct an imaginary run that helps eventually deduce valuable information about correct processes in the system and emulate $\diamond \mathcal{S}$.

$? \mathcal{P}+\diamond \mathcal{S}$ is shown to be the weakest failure detector within $\mathcal{A}$ to solve NBAC with a majority of correct processes. As a corollary of our result, we show that in a system equipped with timeless failure detectors, NBAC is strictly harder than Consensus. Roughly speaking, in the class $\mathcal{A}$ of timeless failure detectors, the difference between the problems is exactly captured by ?P .

Roadmap. Section 2 defines our system model. Section 3 presents the class $\mathcal{A}$ of timeless failure detectors. Section 4 gives a brief reminder of the technique of [1] and discusses its applicability to the NBAC problem. Section 5 proves formally that, within $\mathcal{A}, \diamond \mathcal{S}$ is necessary to solve NBAC. Section 5 draws 
as corollaries that $\diamond \mathcal{S}+? \mathcal{P}$ is the weakest failure detector to solve NBAC and that NBAC is strictly harder than Consensus in $\mathcal{A}$. Section 6 concludes the paper by discussing some related work.

\section{Model}

We consider in this paper a crash-prone asynchronous message passing model augmented with the failure detector abstraction. We recall here what in the model is needed to state and prove our results. More details on the model can be found in [2].

System. We assume the existence of a global clock to simplify the presentation. This is actually a fictional device: the processes do not have direct access to it (timing assumptions are captured within failure detectors). We take the range $\mathcal{T}$ of the clock output values to be the positive real axis: $[0,+\infty)$. We consider an asynchronous distributed system in which there is no time bound on message delay, clock drift, or the time necessary to execute a step [3]. The system consists of a set of $n$ processes $\Pi=\left\{p_{1}, . ., p_{n}\right\}(n>0)$. Every pair of processes is connected by a reliable communication channel.

Failures and failure patterns. Processes are subject to crash failures. A failure pattern $F$ is a function from the global time range $\mathcal{T}$ to $2^{\Pi}$, where $F(t)$ denotes the set of processes that have crashed by time $t$. Once a process crashes, it does not recover, i.e., $\forall t<t^{\prime}: F(t) \subseteq F\left(t^{\prime}\right)$. We define $\operatorname{correct}(F)=$ $\Pi-\cup_{t \in \mathcal{T}} F(t)$ to be the set of correct processes. A process $p \notin F(t)$ is said to be $u p$ at time $t$. A process $p \in F(t)$ is said to be crashed (or incorrect) at time $t$. We do not consider Byzantine failures: a process either correctly executes the algorithm assigned to it, or crashes and stops executing any action forever. An environment $\mathcal{E}$ is a set of possible failure patterns. By default, we consider here environments of the form $\mathcal{E}_{f}$ in which up to $f$ processes can fail. We assume that there is at least one correct process: $0 \leq f<n$.

Failure detectors. A failure detector history $H$ with range $\mathcal{R}$ is a function from $\Pi \times \mathcal{T}$ to $\mathcal{R}$. $H(p, t)$ is the output of the failure detector module of process $p$ at time $t$. A failure detector $\mathcal{D}$ is a function that maps each failure pattern $F$ to a set of failure detector histories $\mathcal{D}(F)$ with range $\mathcal{R}_{\mathcal{D}} \cdot \mathcal{D}(F)$ denotes the set of possible failure detector histories permitted by $\mathcal{D}$ for the failure pattern F.

Every process $p_{i}$ has a failure detector module $\mathcal{D}_{i}$ that $p_{i}$ queries to obtain information about the failures in the system. Typically, this information includes the set of processes that a process currently suspects to have crashed. ${ }^{1}$

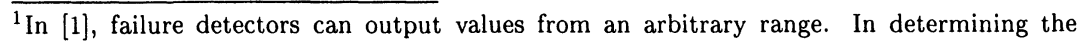
weakest failure detector for NBAC, we do not make any assumption a priori on the range of a failure detector.
} 
Among the failure detectors defined in [2], we consider the following ones, each defined by a completeness and an accuracy property:

Perfect $(\mathcal{P})$ : strong completeness (i.e., every incorrect process is eventually suspected by every correct process) and strong accuracy (i.e., no process is suspected before it crashes);

Eventually strong $(\diamond \mathcal{S})$ : strong completeness and eventual weak accuracy (i.e., there is a time after which one correct process is never suspected).

For any failure pattern $F, \mathcal{P}(F), \diamond \mathcal{S}(F)$ and ?P $(F)$ denote the sets of all histories satisfying the corresponding properties.

Algorithms. We model the set of asynchronous communication channels as a message buffer which contains messages not yet received by their destinations. An algorithm $A$ is a collection of $n$ (possibly infinite state) deterministic automata, one for each of the processes. $A\left(p_{i}\right)$ denotes the automaton running on process $p_{i}$. In each step of $\mathrm{A}$, process $p_{i}$ performs atomically the following three actions: (1) $p_{i}$ chooses non-deterministically a single message addressed to $p_{i}$ from the message buffer, or a null message, denoted $\lambda$; (2) $p_{i}$ queries and receives a value from its failure detector module; (3) $p_{i}$ changes its state and sends a message to a single process according to the automaton $A\left(p_{i}\right)$, based on its state at the beginning of the step, the message received in the receive action, and the value that $p_{i}$ sees in the failure detector query action.

Configurations, schedules and runs. A configuration defines the current state of each process in the system and the set of messages currently in the message buffer. Initially, the message buffer is empty. A step $\left(p_{i}, m, d, A\right)$ of an algorithm $A$ is uniquely determined by the identity of the process $p_{i}$ that takes the step, the message $m$ received by $p_{i}$ during the step ( $m$ might be the null message $\lambda$ ), and the failure detector value $d$ seen by $p_{i}$ during the step. We say that a step $e=\left(p_{i}, m, d, A\right)$ is applicable to the current configuration if and only if $m=\lambda$ or $m$ is a message from the current message buffer destined to $p_{i}$. $e(C)$ denotes the unique configuration that results when $e$ is applied to $C$. A schedule $S$ of algorithm $A$ is a (finite or infinite) sequence of steps of $A$. $S_{\text {T }}$ denotes the empty schedule. We say that a schedule $S$ is applicable to a configuration $C$ if and only if (a) $S=S_{\mathrm{T}}$, or (b) $S$ [1] is applicable to $C, S[2]$ is applicable to $S[1](C)$, etc. For a finite schedule $S$ applicable to $C, S(C)$ denotes the unique configuration that results from applying $S$ to $C$.

A partial run of algorithm $A$ in an environment $\mathcal{E}$ using a failure detector $\mathcal{D}$ is a tuple $R=\left\langle F, H_{\mathcal{D}}, I, S, T\right\rangle$ where $F \in \mathcal{E}$ is a failure pattern, $H_{\mathcal{D}} \in \mathcal{D}(F)$ is a failure detector history, $I$ is an initial configuration of $A, S$ is a finite schedule of $A$, and $T \subseteq \mathcal{T}$ is a finite list of increasing time values (indicating when each step $S$ occurred) such that $|S|=|T|, S$ is applicable to $I$, and for all $t \leq|S|$, if $S[t]$ is of the form $\left(p_{i}, m, d, A\right)$ then: (1) $p_{i}$ has not crashed by time $T[t]$, i.e., $p_{i} \notin F(T[t])$ and (2) $d$ is the value of the failure detector module $\mathcal{D}_{i}$ at time $T[t]$, i.e., $d=H_{\mathcal{D}}\left(p_{i}, T[t]\right)$. 
A run of algorithm $A$ in an environment $\mathcal{E}$ using a failure detector $\mathcal{D}$ is a tuple $R=\left\langle F, H_{\mathcal{D}}, I, S, T\right\rangle$, where $S$ is an infinite schedule of $A$ and $T \subseteq \mathcal{T}$ is an infinite list of increasing time values indicating when each step $S$ occurred. In addition to satisfying the properties (1) and (2) of a partial run, run $R$ should guarantee that (3) every correct process in $F$ takes an infinite number of steps in $S$ and eventually receives every message sent to it (this conveys the reliability of the communication channels).

Weakest failure detector. A problem is a set of runs (usually defined by a set of properties that these runs should satisfy). We say that a failure detector $\mathcal{D}$ solves problem $M$ in an environment $\mathcal{E}$ if there is an algorithm $A$, such that all the runs of $A$ in $\mathcal{E}$ using $\mathcal{D}$ are in $M$ (i.e., they satisfy the properties of $M$ ).

Let $\mathcal{D}$ and $\mathcal{D}^{\prime}$ be any two failure detectors and $\mathcal{E}$ be any environment. If there is an algorithm $T_{\mathcal{D}^{\prime} \rightarrow \mathcal{D}}$ that emulates $\mathcal{D}$ with $\mathcal{D}^{\prime}$ in $\mathcal{E}\left(T_{\mathcal{D}^{\prime} \rightarrow \mathcal{D}}\right.$ is called a reduction algorithm), we say that $\mathcal{D}$ is weaker than $\mathcal{D}^{\prime}$ in $\mathcal{E}$, or $\mathcal{D} \preceq_{\mathcal{E}} \mathcal{D}^{\prime}$. If $\mathcal{D} \preceq_{\mathcal{E}} \mathcal{D}^{\prime}$ but $\mathcal{D}^{\prime} \swarrow_{\mathcal{E}} \mathcal{D}$ we say that $\mathcal{D}$ is strictly weaker than $\mathcal{D}^{\prime}$ in $\mathcal{E}$, or $\mathcal{D} \prec \mathcal{E} \mathcal{D}^{\prime} .^{2}$ Note that $T_{\mathcal{D}^{\prime} \rightarrow \mathcal{D}}$ does not need to emulate all histories of $\mathcal{D}$; it is required that all the failure detector histories it emulates be histories of $\mathcal{D}$.

We say that a failure detector $\mathcal{D}$ is the weakest failure detector to solve a problem $M$ in environment $\mathcal{E}$ if two conditions are satisfied: (1) Sufficiency: $\mathcal{D}$ solves $M$ in $\mathcal{E}$, and (2) Necessity: if a failure detector $\mathcal{D}^{\prime}$ solves $M$ in $\mathcal{E}$ then $\mathcal{D} \preceq \mathcal{E} \mathcal{D}^{\prime}$

We say that problem $M$ is harder than problem $M^{\prime}$ in environment $\mathcal{E}$, if any failure detector $\mathcal{D}$ solving $M$ in $\mathcal{E}$ solves also $M^{\prime}$ in $\mathcal{E}$. Respectively, $M$ is strictly harder than $M^{\prime}$ in $\mathcal{E}$, if $M$ is harder than $M^{\prime}$ in $\mathcal{E}$ and there exists a failure detector $\mathcal{D}^{\prime}$ that solves $M^{\prime}$ (in $\mathcal{E}$ ) but not $M$.

\section{Timeless failure detectors}

This section introduces a new class $\mathcal{A}$ of timeless failure detectors. Intuitively, a timeless failure detector module is not able to provide information about when exactly (in the sense of the global time) failures have occurred.

We denote by $F_{0}$ the failure-free failure pattern: $\forall t \in \mathcal{T}, F_{0}(t)=\varnothing$. For any $F \in \mathcal{E}_{f}$ and $\delta \in \mathcal{T}$, we introduce the failure pattern $F_{\delta}$, such that, for all $t \in \mathcal{T}$ :

$$
F_{\delta}(t)=\left\{\begin{array}{rll}
\varnothing & \text { if } & t<\delta \\
F(t-\delta) & \text { if } & t \geq \delta
\end{array}\right.
$$

Thus, for a failure that occurs at time $t$ in $F$, the corresponding failure in $F_{\delta}$ occurs at $t+\delta$, and no failure occurs before time $\delta$ in $F_{\delta}$. Note that $\forall \delta \in \mathcal{T}$ : $\operatorname{correct}(F)=\operatorname{correct}\left(F_{\delta}\right)$. That is, a process is correct in $F$ if and only if it is correct in $F_{\delta}$. Thus, for any $f$, if $F \in \mathcal{E}_{f}$, then $F_{\delta} \in \mathcal{E}_{f}$.

\footnotetext{
${ }^{2}$ Later we omit $\mathcal{E}$ in $\prec \mathcal{E}$ and $\preceq \mathcal{E}$ when there is no ambiguity on the environment $\mathcal{E}$.
} 
Formally, the class $\mathcal{A}$ consists of all (timeless) failure detectors $\mathcal{D}$, such that:

$$
\begin{aligned}
& \exists H_{0} \in \mathcal{D}\left(F_{0}\right), \forall \delta \in \mathcal{T} \\
& \forall F \in \mathcal{E}_{f}, \forall H \in \mathcal{D}(F), \\
& \exists H_{\delta} \in \mathcal{D}\left(F_{\delta}\right): \forall p_{i} \in \Pi, \forall t \in \mathcal{T}, \\
& H_{\delta}\left(p_{i}, t\right)=\left\{\begin{aligned}
H_{0}\left(p_{i}, t\right) & \text { if } t<\delta \\
H\left(p_{i}, t-\delta\right) & \text { if } t \geq \delta
\end{aligned}\right.
\end{aligned}
$$

It follows from (1) that, for any failure detector $\mathcal{D} \in \mathcal{A}$ and $\delta \in \mathcal{T}$, if a failure occurred at time $t_{0}$ and is reported by a module of $\mathcal{D}$ at time $t_{1}$, then a failure that occurred at $t_{0}+\delta$ could be reported in the same way at $t_{1}+\delta$ : the process does not know when exactly the failure occurred. This captures our idea that failure detectors of class $\mathcal{A}$ provide no information about the time when failures occur.

We restrict our scope from the original universe of failure detectors [2] to

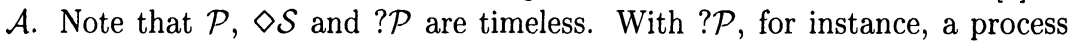
can detect the very fact that some process has crashed, but there is no way to acquire the actual time at which the failure occurred.

Examples of histories output by a failure detector $\mathcal{D}$ from $\mathcal{A}$ are depicted in Figure 1. The system consists of two processes $p_{1}$ and $p_{2}$. At any time, $\mathcal{D}_{i}$ outputs a set of processes suspected by $p_{i}(i=1,2)$. Assume that (a) $p_{2}$ crashes at time $t_{1}$ and $p_{1}$ detects the failure at time $t_{2}$. Then, by the definition of $\mathcal{A}$, if (b) $p_{2}$ crashes at time $t_{1}+d$, then there exists a history of $\mathcal{D}$ in which $p_{1}$ detects the failure at time $t_{2}+d$.

(a)

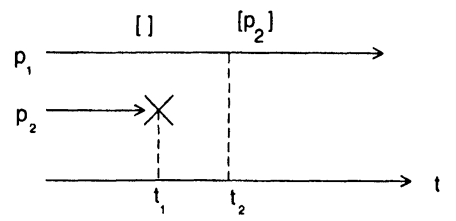

(b)

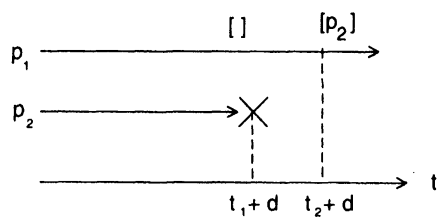

Figure 1. Examples of timeless failure detector histories.

Consider the failure detector $\mathcal{B}[\alpha]$ mentioned in the introduction: each module of $\mathcal{B}[\alpha]$ outputs $T$ or a subset of the processes in $\Pi\left(\mathcal{R}_{\mathcal{B}[\alpha]}=T \cup 2^{\Pi}\right)$. Formally, $\mathcal{B}[\alpha]$ is defined as follows:

$$
\begin{aligned}
& \forall F \in \mathcal{E}_{f}, \forall H \in B[\alpha](F), \\
& \exists H_{\mathcal{P}} \in \mathcal{P}(F), \\
& \forall p_{i} \in \Pi, \exists t_{i} \in \mathcal{T}, \forall t \in \mathcal{T}: \\
& H\left(p_{i}, t\right)=\left\{\begin{aligned}
\mathcal{T} & \text { if } t<t_{i} \\
p_{i} & \text { if } t \geq t_{i} \wedge F(\alpha) \neq \varnothing \\
H_{\mathcal{P}}\left(p_{i}, t\right) & \text { if } t \geq t_{i} \wedge F(\alpha)=\varnothing
\end{aligned}\right.
\end{aligned}
$$

(The Stillborn failure detector $\mathcal{B}$ is a particular case of $\mathcal{B}[\alpha]$ with $\alpha=0$ and $t_{i}=0, \forall p_{i} \in \Pi$.) Clearly, $\mathcal{B}[\alpha]$ does not belong to $\mathcal{A}$. Indeed, consider a failure 
pattern $F$ in which only one process is crashed at time $\alpha$. Take a corresponding history $H \in B[\alpha](F)$. For every process $p_{i}$, there is a time $t_{i}$, such that, for any $t \geq t_{i}, H\left(p_{i}, t\right)=p_{i}$. Now consider a failure pattern $F_{\delta}$ in which no process is crashed at time $\alpha$ (the condition holds for all $\delta>\alpha$ ). Since failure detector module of $p_{i}$ behaves now like $\mathcal{P}_{i}$ (i.e., the perfect failure detector), its own identity $p_{i}$ is never output. Thus, $\mathcal{B}[\alpha] \notin \mathcal{A}$.

\section{Proof technique}

Before proving that, in $\mathcal{A}, \diamond \mathcal{S}$ is necessary to solve NBAC, we briefly recall here the technique used in [1] to prove that $\diamond \mathcal{S}$ is necessary to solve Consensus and we discuss the applicablity of this technique to NBAC.

The weakest failure detector to solve Consensus. A failure detector, denoted $\Omega(\diamond \mathcal{S} \preceq \Omega)$ is defined in [1]. The output of $\Omega_{i}$ is a single process $p_{j}$, that $p_{i}$ currently trusts, i.e., that $p_{i}$ considers to be correct $\left(\mathcal{R}_{\Omega}=\right.$ $\Pi)$. For each failure pattern $F, \Omega(F)$ is the set of all failure detector histories $H_{\Omega}$, such that there is a time after which all correct processes always trust the same correct process. Clearly, $\Omega \in \mathcal{A}$.

Let $\mathcal{E}$ be any environment, $\mathcal{D}$ be any failure detector that solves Consensus in $\mathcal{E}$, and Consensus $_{\mathcal{D}}$ be any Consensus algorithm that uses $\mathcal{D}$. The algorithm $T_{\mathcal{D} \rightarrow \Omega}$ that transforms $\mathcal{D}$ into $\Omega$ in $\mathcal{E}$ works as follows.

Fix an arbitrary run of $T_{\mathcal{D} \rightarrow \Omega}$ using $\mathcal{D}$, with failure pattern $F \in \mathcal{E}$ and failure detector history $H_{\mathcal{D}} \in \mathcal{D}(F)$. All processes periodically query their failure detector $\mathcal{D}$ and exchange information about the values of $H_{\mathcal{D}}$ that they see in this run. Using this information, the processes construct a directed acyclic graph (DAG) that represents a "sampling" of failure detector values in $H_{\mathcal{D}}$ and causal relationships between the values. By periodically sending its current version of the DAG to all processes, and incorporating all the DAGs that it receives into its own DAG, every correct process constructs ever increasing finite approximations of the same infinite limit DAG $G$.

The DAG $G$ can be used to simulate runs of Consensus $_{\mathcal{D}}$ with failure pattern $F$ and failure detector history $H_{\mathcal{D}}$. These runs could have happened if processes were running Consensus $_{\mathcal{D}}$ instead of $T_{\mathcal{D} \rightarrow \Omega}$. If we simulate all possible runs of Consensus $_{\mathcal{D}}$ applied to the DAG $G$ with all possible initial configurations $I$, we obtain a simulation forest: a tree for each initial configuration.

Thus, the infinite DAG $G$ induces an infinite simulation forest $\Upsilon$ of runs of Consensus $_{\mathcal{D}}$ with failure pattern $F$ and failure detector history $H_{\mathcal{D}} \in \mathcal{D}(F)$. From the properties of the Consensus problem, it follows that $\Upsilon$ comprises schedules corresponding to the runs of Consensus in which every correct process decides 0 and runs in which every correct process decides 1 . This allows to design a deterministic algorithm that identifies a process $p^{*}$ that is correct in $F$, namely a process whose step defines which decision is going to be taken by the rest of correct processes in the descending schedules.

Although the simulation forest $\Upsilon$ is infinite and cannot be computed by any process, there exists a finite subforest of $\Upsilon$ that gives sufficient information to 
identify $p^{*}$. Thus, there is a time after which, every correct process running $T_{\mathcal{D} \rightarrow \Omega}$ obtains a reference $p^{*}$. In other words, $T_{\mathcal{D} \rightarrow \Omega}$ emulates $\Omega$.

NBAC: a hard nut. As we discussed for $\diamond \mathcal{S}, \Omega$ is not at all necessary to solve NBAC (as shown in [6]). Thus, the technique of [1] cannot be directly applied to show the necessity of $\Omega$ for NBAC. For instance, if a module of the stillborn failure detector $\mathcal{B}$ outputs $T$, then there is an initial failure in the system and NBAC is trivially solved by deciding abort at every correct process. There is no way to identify correct processes and, thus, no algorithm $T_{\mathcal{B} \rightarrow \Omega}$ is possible.

However, even if we exclude failure detectors like $\mathcal{B}[\alpha]$ by considering timeless failure detectors only (i.e., focusing on class $\mathcal{A}$ ), we are still not able to apply the technique of [1]. Indeed, let $\mathcal{D}$ be any failure detector that solves $\mathrm{NBAC}$ in an environment $\mathcal{E}$. Consider a run of an NBAC algorithm using $\mathcal{D}$ in a failure pattern $F$, such that $F(0) \neq \varnothing$ (some process is initially crashed). Clearly, no process can decide commit (no matter which failure detector history $H_{\mathcal{D}}$ is output by $\mathcal{D}$ ). The only decision a correct process can take is abort (otherwise, the A-Validity property of NBAC would be violated, since some $p \in F(0)$ could have voted $n o$ ). In this case, the corresponding simulation forest $\Upsilon$ does not bring any valuable information about failures to identify a correct process (no matter which failure detector history $H_{\mathcal{D}}$ is output by $\mathcal{D}$ ).

Fortunately, thanks to the very nature of timeless failure detectors, we can modify the original DAG $G$ in order to fetch a valuable information about correct processes of $F$. The details are presented in Section 5 .

\section{Necessary condition}

This section shows the necessity of $\Omega$ to solve NBAC using failure detectors in class $\mathcal{A}$. To this end, we present a reduction algorithm $T_{\mathcal{D} \rightarrow \Omega}$ transforming any failure detector $\mathcal{D} \in \mathcal{A}$ that solves NBAC into $\Omega$. A corollary of our result is that $\diamond \mathcal{S}$ (which is weaker than $\Omega$ ) is necessary to solve NBAC (using $\mathcal{A}$ ), and hence $? \mathcal{P}+\diamond \mathcal{S}$ is the weakest failure detector within $\mathcal{A}$ to solve NBAC.

Nice runs and nice DAGs. Let $\mathcal{D}$ be any failure detector in $\mathcal{A}$ and $N B A C_{\mathcal{D}}$ be any NBAC algorithm using $\mathcal{D}$. From now on, we denote by $e=$ $(p, m, d)$ a step of process $p$ executing $N B A C_{\mathcal{D}}$.

Let $F_{0}$ be the failure-free pattern and $H_{0}$ be the history from $\mathcal{D}\left(F_{0}\right)$, such that the condition (1) in Section 3 for $\mathcal{D}$ holds with $H_{0}$. Let $I$ be any initial configuration in which all processes vote yes. Due to the properties of NBAC, there exists a partial run $R_{0}=\left\langle F_{0}, H_{0}, I, S_{0}, T_{0}\right\rangle$ of $N B A C_{\mathcal{D}}$ comprising a finite number of steps in which every process decides commit.

Taking the nice run $R_{0}$ as a basis, we can now construct a nice DAG (directed acyclic graph) $G_{0}$ induced by the failure-free pattern $F_{0}$. For any step $e=(p, m, d)$ in $S_{0}$, we create a vertex $[p, d, k]$ of $G_{0}$, where $k-1$ is the number of steps of $p$ in $S_{0}$ preceding $e$. For any steps $e_{1}=\left(p_{1}, m_{1}, d_{1}\right)$ and $e_{2}=\left(p_{2}, m_{2}, d_{2}\right)$ in $S_{0}$, such that $e_{1}$ precedes $e_{2}$ in $S_{0}$, we create a correspond- 
ing edge in $\left[p_{1}, d_{1}, k_{1}\right] \rightarrow\left[p_{2}, d_{2}, k_{2}\right]$ in $G_{0}$. This means that $p_{2}$ queried its failure detector for the $k_{2}$-th time after $p_{1}$ queried its failure detector for the $k_{1}$-th time.

Constructing a DAG. Let $F$ be any failure pattern from $\mathcal{E}_{f}$ and $H \in$ $\mathcal{D}(F)$ and assume that the same nice DAG $G_{0}$ is initially available to all processes. Consider a run $R$ of $T_{\mathcal{D} \rightarrow \Omega}$. Processes periodically query their failure detector $\mathcal{D}$ and exchange information about the values of $H \in \mathcal{D}(F)$ that they see in the current run. Using this information, every process $p$ constructs an imaginary DAG $G_{p}$, in which the real samples of $H$ are assumed to be seen after all the values of $H_{0}$ presented in $G_{0}$. That is, every time a process $p$ sees a failure detector value $d,(1)$ a new vertex $[p, d, k]$ is added to $G_{p}$, such that $k=k_{0}+k_{R}$, where $k_{0}$ is the number of steps of $p$ in $S_{0}$ and $k_{R}$ is the number of times $p$ queried its failure detector module so far, and (2) a new edge from every vertex of $G_{p}$ to $[p, d, k]$ is added. As a result, every correct process $p$ maintains an ever growing graph $G_{p}(t)$, such that $G_{p}(t) \rightarrow_{t \rightarrow \infty} G$ for some infinite DAG $G$. Note that $G$ contains a sampling of the failure detector history $H$ corresponding to a real failure pattern $F(H \in \mathcal{D}(F)$ as well as of some imaginary history $H_{0} \in \mathcal{D}\left(F_{0}\right)$, where $F_{0}$ is the failure-free pattern.

We say that an infinite DAG $\tilde{G}$ is a sampling $D A G$ of history $\tilde{H} \in \mathcal{D}(\tilde{F})$ if the following properties are satisfied:

1 The vertices of $\tilde{G}$ are of the form $[p, d, k]$ where $d=\tilde{H}(p, t)$ for some $t$ and $k \in \mathbb{N}$.

2 If $\left[p_{1}, d_{1}, k_{1}\right] \rightarrow\left[p_{2}, d_{2}, k_{2}\right]$ is an edge of $\tilde{G}$ and $d_{1}=\tilde{H}\left(p_{1}, t_{1}\right)$ and $d_{2}=$ $\tilde{H}\left(p_{2}, t_{2}\right)$, then $t_{1}<t_{2}$.

3 If $[p, d, k],\left[p, d^{\prime}, k^{\prime}\right]$ are vertices of $\tilde{G}$ and $k<k^{\prime}$ then $[p, d, k] \rightarrow\left[p, d^{\prime}, k^{\prime}\right]$ is an edge of $\tilde{G}$.

$4 \tilde{G}$ is transitively closed.

5 Let $V$ be any finite subset of vertices of $\tilde{G}$ and $p$ be any correct process. There is $d \in \mathcal{R}_{\mathcal{D}}$ and $k \in \mathbb{N}$, such that for every vertex $v \in V, v \rightarrow[p, d, k]$ is an edge of $\tilde{G}$.

The following lemma precisely captures the relationship between the real failure pattern $F$ and the DAG $G$ constructed by $T_{\mathcal{D} \rightarrow \Omega}$ (the proof is omitted due to the space limitations and it can be found in [7]):

Lemma 1 There exists $\delta \in \mathcal{T}$ and a failure detector history $H_{\delta} \in \mathcal{D}\left(F_{\delta}\right)$ such that $G$ is a sampling $D A G$ of $H_{\delta}$.

Thus, $G$ represents a sample of a failure detector history $H_{\delta}$ that could be seen if the failure pattern was $F_{\delta}$. Note that even if a process $p$ is initially crashed in $F, G$ contains the samples of its failure detector module output. However, the number of vertices of the form $[p, \cdot, \cdot] \in G$ is finite, thus, $p$ cannot be considered 
to be correct in $F_{\delta}$. In other words, a crashed process in $F$ cannot appear to be correct in $F_{\delta}$.

Tags and decision gadgets. Lemma 1 allows us to use $G$ to simulate some of the runs of $N B A C_{\mathcal{D}}$ in the failure pattern $F_{\delta}$. Take an initial configuration $I$ of $N B A C_{\mathcal{D}}$ in which every process votes yes. The set of simulated schedules of $N B A C_{\mathcal{D}}$ that are compatible with some path of $G$ and are applicable to $I$ can be organized as a tree $\Upsilon$ : paths in this tree represent simulated schedules of $N B A C_{\mathcal{D}}$ with initial configuration $I$. The fact that $G_{0} \subset G$ guarantees that there exists a schedule in $\Upsilon$ in which every process decides commit.

Following [1], we assign a set of tags (abort or commit) to each vertex of the simulation tree $\Upsilon$ induced by $G$. Vertex $S$ of tree $\Upsilon$ gets tag $k$ if and only if it has a descendant $S^{\prime}$ (possibly $S=S^{\prime}$ ) such that some correct process has decided $k$ in $S^{\prime}(I)$. A vertex of $\Upsilon$ is monovalent if it has only one tag, and bivalent if it has both tags (following the terminology of [3]).

Still following [1], we also introduce the notion of decision gadgets and deciding processes and show that any deciding process in $\Upsilon$ is correct. Informally, a decision gadget is a vertex $S$ of $\Upsilon$ having exactly two monovalent leaves: one abort-valent and one commit-valent. In turn, a deciding process of $S$ is a process whose step defines the decision taken by a descendant of $S$. The following lemma gives a condition of the existence of at least one decision gadget in $\Upsilon$ :

Lemma 2 If $\operatorname{correct}(F) \neq \Pi$ ( $F$ is not failure-free), then $\Upsilon$ has a decision gadget.

Proof: Let $p \notin \operatorname{correct}(F)$. There exists a finite schedule $E$ in $\Upsilon$ containing only steps of correct processes such that all correct processes have decided in in $E(I)$ (Lemma 10 of [1]). Since $E$ contains no step of process $p$, no information is available about its initial vote, and the decision value must be abort (otherwise the A-Validity property is violated). From the way the simulation tree is constructed, it follows that $\Upsilon$ contains a schedule in which commit is decided. Thus the initial configuration of $\Upsilon$ is bivalent. By Lemma 18 of [1], $\Upsilon$ has at least one decision gadget (and hence a deciding process).

Reduction algorithm. Now we are ready to define a rule according to which the same correct process is eventually identified by every correct process. Every process $p$ periodically updates and tags a simulation tree $\Upsilon_{p}$ induced by $G_{p}$ with the initial configuration $I$ in which all processes vote yes. If there exists a decision gadget in $\Upsilon_{p}$, then $T_{\mathcal{D} \rightarrow \Omega}$ outputs the deciding process of the smallest decision gadget of $\Upsilon_{p}$ (since the set of vertices of $\Upsilon_{p}$ is countable, we can easily impose a rule to define the smallest decision gadget in it), otherwise $T_{\mathcal{D} \rightarrow \Omega}$ outputs $p_{1}$. Note that for any correct process $p, G=\lim _{t \rightarrow \infty} G_{p}(t)$ and thus $\Upsilon=\lim _{t \rightarrow \infty} \Upsilon_{p}(t)$. 
Theorem 3 There exists a process $p^{*} \in \operatorname{correct}(F)$, such that, for every correct process $p$, there is a time after which $T_{\mathcal{D} \rightarrow \Omega}$ outputs $p^{*}$, forever.

Proof: Consider $\delta \in \mathcal{T}$ and failure pattern $F_{\delta}$, such that $G$ is a sampling DAG for some $H_{\delta} \in \mathcal{D}\left(F_{\delta}\right)$. Note that $\operatorname{correct}(F)=\operatorname{correct}\left(F_{\delta}\right)$. Two cases are possible:

(1) $F$, and thus $F_{\delta}$ are failure-free. Then all vertices in $\Upsilon$ are monovalent and the reduction algorithm forever outputs $p_{1} \in \operatorname{correct}(F)$.

(2) $F$, and thus $F_{\delta}$ are not failure-free. By Lemma $2, \Upsilon$ has a deciding process. Let $p^{*}$ be the deciding process of the smallest decision gadget. Since ever growing simulation trees $\Upsilon_{p}(t)$ of all correct processes $p$ tend to $\Upsilon$, there exists $t_{0}$ such that $\forall t>t_{0}, \forall p \in \operatorname{correct}(F)$, the decision process of the smallest decision gadget is $p^{*}$. Thus, $\forall t>t_{0}$ all correct processes $p$ have output $p_{p}=p^{*}$. By Lemma 21 of [1], the deciding process is correct in $F$. Thus $T_{\mathcal{D} \rightarrow \Omega}$ outputs the identity of a correct process.

Theorem 4 For any environment $\mathcal{E}_{f}$, if a failure detector $\mathcal{D} \in \mathcal{A}$ can be used to solve $N B A C$ in $\mathcal{E}_{f}$, then $\mathcal{D} \succeq_{\mathcal{E}_{f}} \Omega$.

From the facts that ?P is necessary to solve NBAC in any environment [6], $\diamond \mathcal{S}$ is weaker than $\Omega$ [1], and Theorem 4, the following result holds:

Theorem 5 For any environment $\mathcal{E}_{f}$, if a failure detector $\mathcal{D} \in \mathcal{A}$ solves $N B A C$ in $\mathcal{E}_{f}$, then $\mathcal{D} \succeq_{\mathcal{E}_{f}}$ ?P $+\diamond \mathcal{S}$.

From the theorem above and [6], we have:

Corollary $6 ? \mathcal{P}+\diamond \mathcal{S}$ is the weakest among timeless failure detectors to solve $N B A C$ in any environment $\mathcal{E}_{f}$ with $f<\left\lceil\frac{n}{2}\right\rceil$.

From [1], [6] and Corollary 6, we have:

Corollary 7 For any environment $\mathcal{E}_{f}$ with $0<f<\left\lceil\frac{n}{2}\right\rceil$ in a system augmented with timeless failure detectors, NBAC is strictly harder than Consensus.

\section{Concluding remarks}

Sabel and Marzullo showed in [8] that $\mathcal{P}$ is the weakest failure detector to solve the Leader Election problem within a specific class of failure detectors. They focus on failure detectors that output sets of suspected processes and satisfy the following symmetry property: if a process detects a failure erroneously, then any process can detect a failure erroneously an arbitrary number of times. The requirement is rather strong: for instance, it excludes all failure detectors that make a finite number of mistakes. The approach is somewhat similar to ours. We also defined a subset $\mathcal{A}$ of the overall universe of failure detectors [2] in which ?P $+\diamond \mathcal{S} \prec \mathcal{P}$ is shown to be the weakest to solve our NBAC problem. 
The class of symmetric failure detectors of [8] and our class $\mathcal{A}$ of timeless failure detectors are however incomparable.

Fromentin, Raynal and Tronel stated in [4] that $\mathcal{P}$ is the weakest failure detector to solve NBAC. Guerraoui [6] pointed out that [4] assumes NBAC to be solved among any subset of the processes in the system and showed that $\mathcal{P}$ is not the weakest failure detector to solve NBAC without that assumption. In this paper, we make a step further showing that a failure detector ?P $+\diamond \mathcal{S} \prec \mathcal{P}$ is the weakest to solve NBAC in a wide class $\mathcal{A}$ of timeless failure detectors (provided an environment with a majority of correct processes). Thus, in this environment, NBAC is strictly harder than Consensus (which is not true in general $[5,6])$. The question of the weakest failure detector to solve NBAC without assuming a majority of correct processes is open for future research.

\section{References}

[1] T. D. Chandra, V. Hadzilacos, and S. Toueg. The weakest failure detector for solving consensus. Journal of the ACM, 43(4):685-722, March 1996.

[2] T. D. Chandra and S. Toueg. Unreliable failure detectors for reliable distributed systems. Journal of the ACM, 43(2):225-267, March 1996.

[3] M. J. Fischer, N. A. Lynch, and M. S. Paterson. Impossibility of distributed consensus with one faulty process. Journal of the ACM, 32(3):374-382, April 1985.

[4] E. Fromentin, M. Raynal, and F. Tronel. On classes of problems in asynchronous distributed systems with process crashes. In Proceedings of the IEEE International Conference on Distributed Systems (ICDCS), pages 470-477, 1999.

[5] R. Guerraoui. On the hardness of failure-sensitive agreement problems. Information Processing Letters, 79(2):99-104, June 2001.

[6] R. Guerraoui. Non-blocking atomic commit in asynchronous distributed systems with failure detectors. Distributed Computing, 15:17-25, January 2002.

[7] R. Guerraoui and P. Kouznetsov. On the weakest failure detector for nonblocking atomic commit. Technical report, School of Computer and Communication Sciences, Swiss Institute of Technology in Lausanne, 2002. Available at http://icwww.epfl.ch/publications/list.php.

[8] L. S. Sabel and K. Marzullo. Election vs. consensus in asynchronous systems. Technical report, Cornell University, Ithaca, NY, TR95-1488, 1995.

[9] D. Skeen. Nonblocking commit protocols. In ACM SIGMOD International Conference on Management of Data, pages 133-142. ACM Press, May 1981. 